\title{
Thermodynamic and Kinetic Aspects of Formamidinium Lead Iodide Thermal Decomposition
}

\author{
Alessio Luongo ${ }^{1}$, Bruno Brunetti ${ }^{2}$, Stefano Vecchio Ciprioti $^{3 *}$, Andrea Ciccioli ${ }^{*}$ and \\ Alessandro Latini ${ }^{1 *}$ \\ ${ }^{1}$ Dipartimento di Chimica, Sapienza Università di Roma, Piazzale Aldo Moro 5, 00185 Roma, Italy \\ ${ }^{2}$ Consiglio Nazionale delle Ricerche - Istituto per lo Studio dei Materiali Nanostrutturati, c/o \\ Dipartimento di Chimica, Sapienza Università di Roma, Piazzale Aldo Moro 5, 00185 Roma, Italy \\ ${ }^{3}$ Dipartimento S.B.A.I, Sapienza Università di Roma, Via del Castro Laurenziano 7, 00161 Roma, \\ Italy \\ *corresponding authors.
}


Table S1. Partial pressures of the main gaseous species estimated by KEMS measurements.

\begin{tabular}{|c|c|c|c|c|c|c|}
\hline & T/K & $\begin{array}{c}\mathbf{p N H}_{3} / \\
\mathbf{P a} \\
\end{array}$ & $\begin{array}{c}\mathbf{p C H}_{4} \mathbf{N}_{2} / \\
\mathbf{P a} \\
\end{array}$ & $\begin{array}{c}\mathbf{p}(\mathbf{H C N})_{3} / \\
\mathbf{P a} \\
\end{array}$ & $\mathbf{p H I} / \mathbf{P a}$ & ртот/ Рa \\
\hline \multirow{3}{*}{$\begin{array}{l}\text { Run } \\
1\end{array}$} & 476 & $\begin{array}{c}8,75^{*} 10^{-} \\
2\end{array}$ & $\begin{array}{c}7,62 * 10^{-} \\
2\end{array}$ & $6,60 * 10^{-8}$ & $\begin{array}{c}1,47 * 10^{-} \\
1\end{array}$ & $\begin{array}{c}3,17 * 10^{-} \\
1\end{array}$ \\
\hline & 488 & $\frac{1,79 * 10^{-}}{1}$ & $\begin{array}{c}1,46 * 10^{-} \\
1\end{array}$ & $2.25 * 10^{-2}$ & $\begin{array}{c}3,95 * 10^{-} \\
1\end{array}$ & $\underset{1}{7,42 * 10^{-}}$ \\
\hline & 513 & $\begin{array}{c}2,98 * 10^{-} \\
1\end{array}$ & $\begin{array}{c}4,62 * 10^{-} \\
1\end{array}$ & $1,16^{*} 10^{-1}$ & $1,50 * 10^{0}$ & $2.37 * 10^{0}$ \\
\hline \multirow{3}{*}{$\begin{array}{c}\text { Run } \\
2\end{array}$} & 513 & $\begin{array}{c}6,75^{*} 10^{-} \\
1\end{array}$ & $\begin{array}{c}5,85 * 10^{-} \\
1\end{array}$ & $1,99 * 10^{-1}$ & $1,86 * 10^{0}$ & $3,31 * 10^{0}$ \\
\hline & 502 & $\begin{array}{c}2,30 * 10 \\
1\end{array}$ & $\begin{array}{c}2,56 * 10^{-} \\
1\end{array}$ & $8,35 * 10^{-2}$ & $\begin{array}{c}7,56 * 10^{-} \\
1\end{array}$ & $1,32 * 10^{0}$ \\
\hline & 488 & $\begin{array}{c}9,46 * 10^{-} \\
2\end{array}$ & $\begin{array}{c}1,17 * 10^{-} \\
1\end{array}$ & $3,15^{*} 10^{-2}$ & $\begin{array}{c}2,95 * 10^{-} \\
1\end{array}$ & $\begin{array}{c}5,38 * 10^{-} \\
1\end{array}$ \\
\hline \multirow{4}{*}{$\begin{array}{c}\text { Run } \\
\quad 3\end{array}$} & 505 & $\begin{array}{c}5,87 * 10^{-} \\
1\end{array}$ & $\begin{array}{c}3,23 * 10^{-} \\
1\end{array}$ & $2,66 * 10^{-1}$ & $1,61 * 10^{0}$ & $2,78^{*} 10^{0}$ \\
\hline & 480 & $\begin{array}{c}1,27 * 10^{-} \\
1\end{array}$ & $\begin{array}{c}7,68 * 10^{-} \\
2\end{array}$ & $7,32 * 10^{-2}$ & $\begin{array}{c}3,33 * 10^{-} \\
1\end{array}$ & $\begin{array}{c}6,10 * 10^{-} \\
-\end{array}$ \\
\hline & 519 & $\begin{array}{c}7,63 * 10^{-} \\
1\end{array}$ & $\begin{array}{c}3,27 * 10^{-} \\
1\end{array}$ & $3,67 * 10^{-1}$ & $2,10^{*} 10^{0}$ & $3,55^{*} 10^{0}$ \\
\hline & 497 & $\begin{array}{c}2,79 * 10^{-} \\
1\end{array}$ & $\begin{array}{c}3,13 * 10^{-} \\
1\end{array}$ & $1,17 * 10^{-1}$ & $\begin{array}{c}6,72 * 10^{-} \\
1\end{array}$ & $1,38 * 10^{0}$ \\
\hline
\end{tabular}

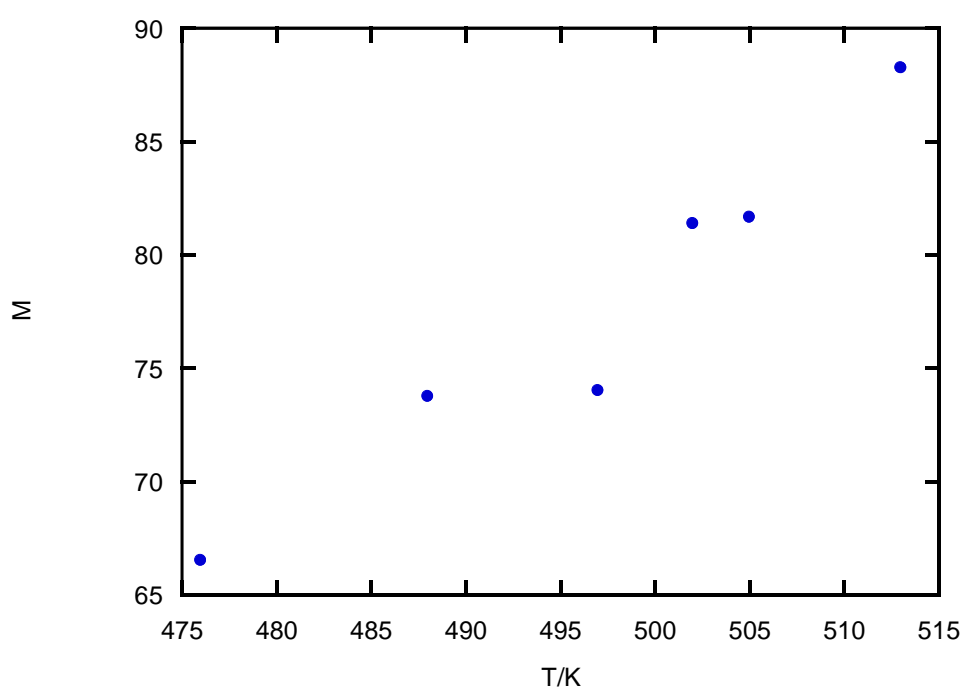

Fig. S2. Mean molecular weight calculated from KEMS spectra. 
Table S2. Total pressures measured by KEML ${ }^{\mathrm{a}}$

\begin{tabular}{|c|c|c|}
\hline Experiment & $\mathbf{T} / \mathbf{K}$ & $\mathbf{P} / \mathbf{P a}$ \\
\hline \multirow{21}{*}{$\operatorname{Exp} 1$} & 452.8 & 0.040 \\
\hline & 448.0 & 0.029 \\
\hline & 443.2 & 0.021 \\
\hline & 438.3 & 0.015 \\
\hline & 433.5 & 0.0099 \\
\hline & 428.4 & 0.0062 \\
\hline & 423.6 & 0.0046 \\
\hline & 418.8 & 0.0028 \\
\hline & 414.0 & 0.0020 \\
\hline & 454.5 & 0.048 \\
\hline & 448.1 & 0.028 \\
\hline & 443.2 & 0.021 \\
\hline & 438.4 & 0.015 \\
\hline & 433.4 & 0.0094 \\
\hline & 428.6 & 0.0060 \\
\hline & 423.6 & 0.0043 \\
\hline & 418.8 & 0.0029 \\
\hline & 413.9 & 0.0019 \\
\hline & 409.0 & 0.0013 \\
\hline & 404.1 & 0.00092 \\
\hline & 398.3 & 0.00053 \\
\hline \multirow[t]{11}{*}{$\operatorname{Exp} 2$} & 457.3 & 0.039 \\
\hline & 452.0 & 0.025 \\
\hline & 446.6 & 0.017 \\
\hline & 441.6 & 0.012 \\
\hline & 436.6 & 0.0085 \\
\hline & 431.8 & 0.0053 \\
\hline & 426.8 & 0.0037 \\
\hline & 422.0 & 0.0026 \\
\hline & 417.2 & 0.0019 \\
\hline & 412.2 & 0.0013 \\
\hline & 407.1 & 0.00083 \\
\hline \multirow[t]{7}{*}{ Exp 3} & 486.8 & 0.25 \\
\hline & 476.5 & 0.14 \\
\hline & 471.9 & 0.11 \\
\hline & 466.8 & 0.076 \\
\hline & 461.8 & 0.056 \\
\hline & 456.9 & 0.041 \\
\hline & 451.9 & 0.027 \\
\hline \multirow[t]{4}{*}{ Exp 4} & 442.8 & 0.020 \\
\hline & 437.6 & 0.015 \\
\hline & 432.7 & 0.0077 \\
\hline & 472.4 & 0.13 \\
\hline
\end{tabular}




\begin{tabular}{|l|l|l|}
\hline & 467.3 & 0.092 \\
\hline & 462.4 & 0.063 \\
\hline & 457.3 & 0.042 \\
\hline & 452.4 & 0.030 \\
\hline & 447.6 & 0.021 \\
\hline & 442.6 & 0.017 \\
\hline & 437.6 & 0.014 \\
\hline
\end{tabular}

${ }^{a}$ In calculating pressures from the mass loss rate, a mean molecular weight of $90 \mathrm{u}$ was assumed (see text) 
Figures S1-S8. JMAK plots in the temperature range $218-235{ }^{\circ} \mathrm{C}$

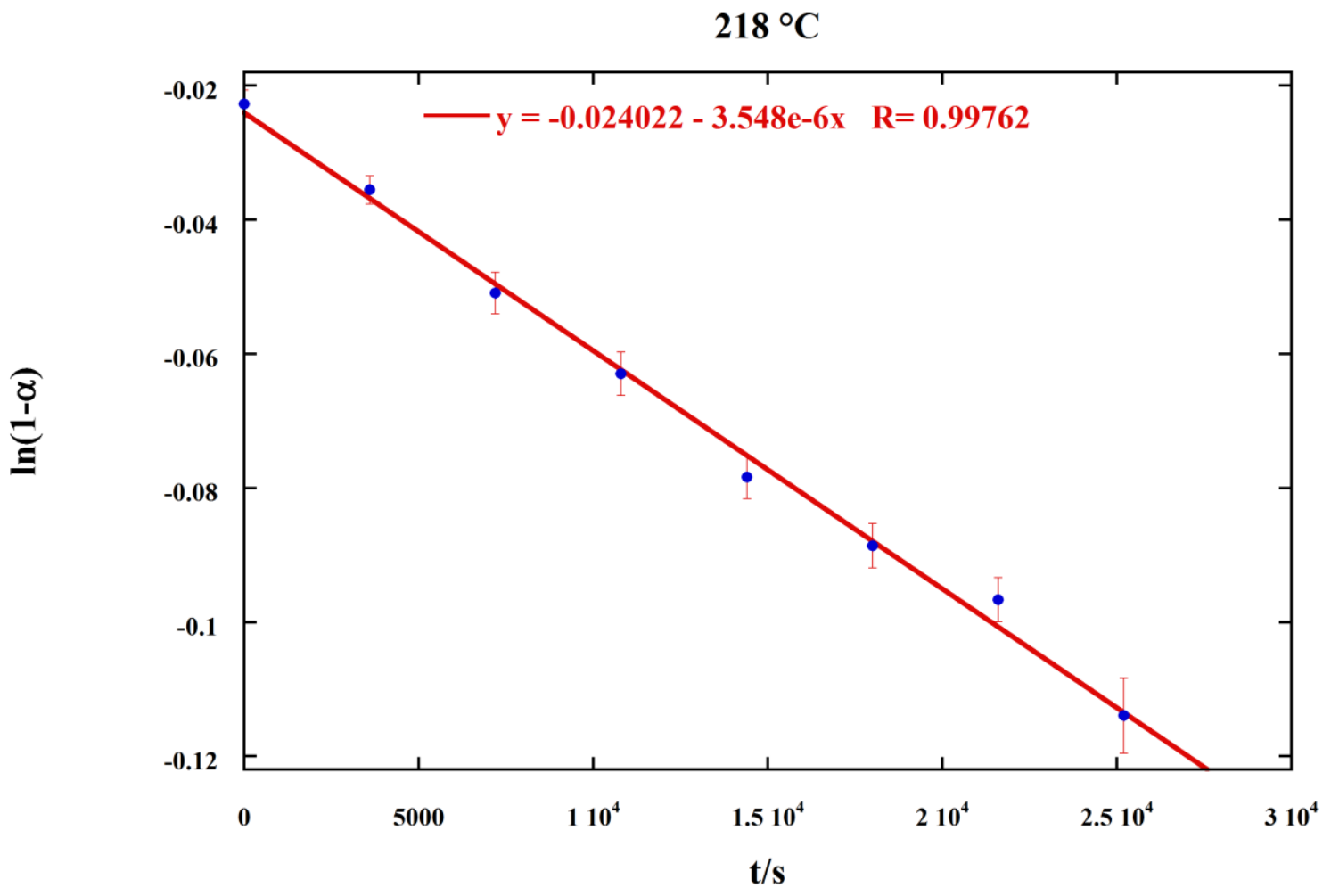

Fig. S1

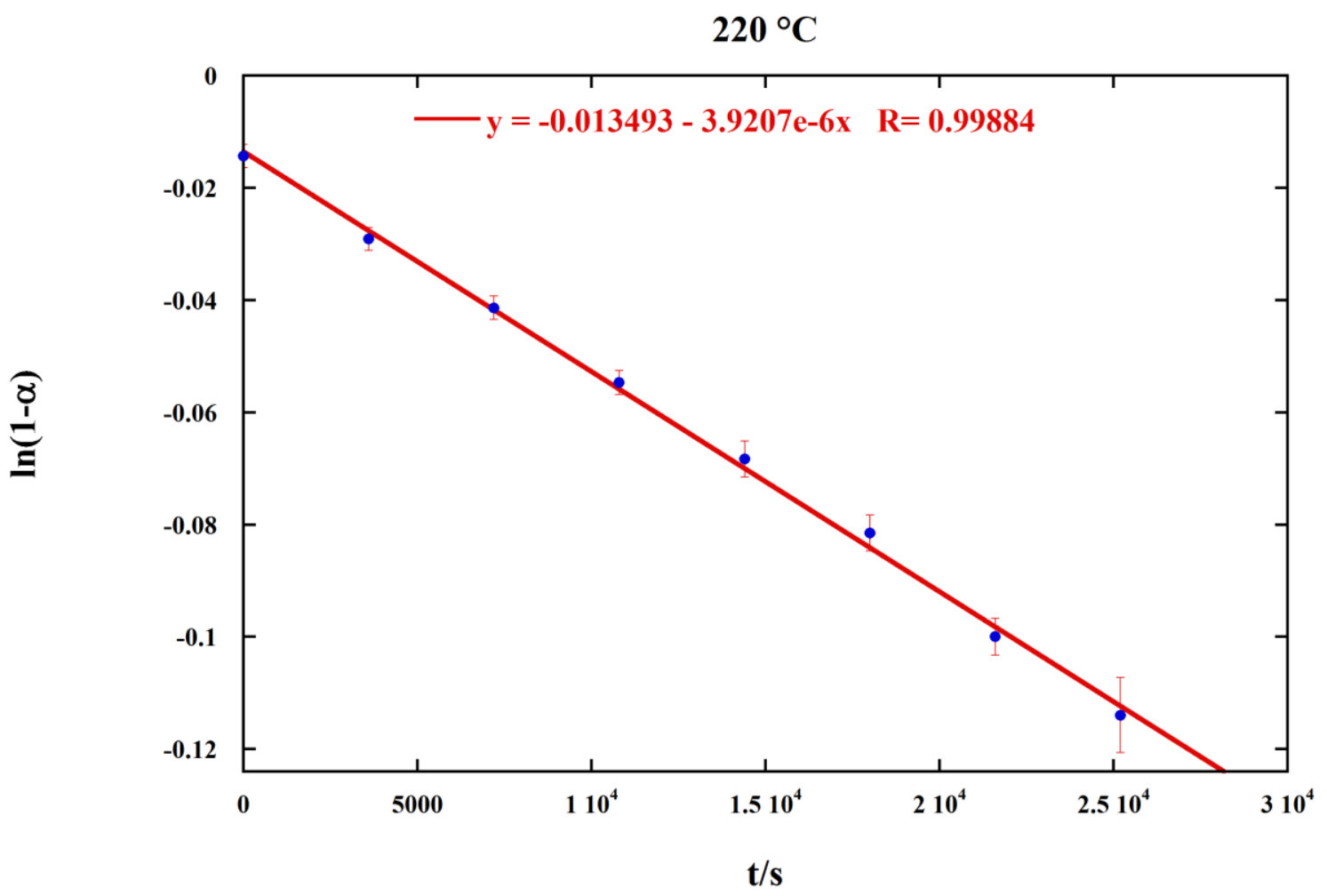

Fig. S2 


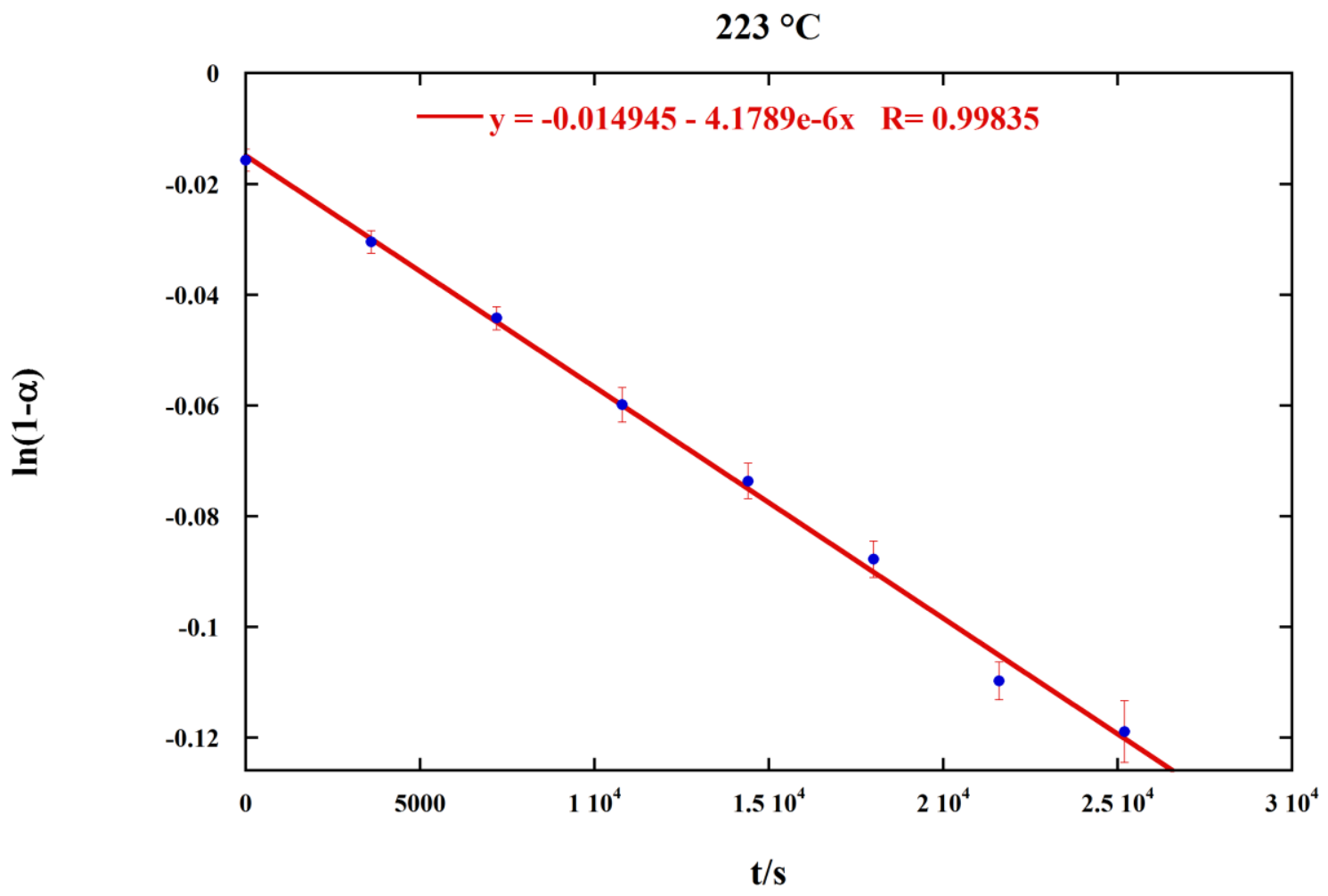

Fig. S3

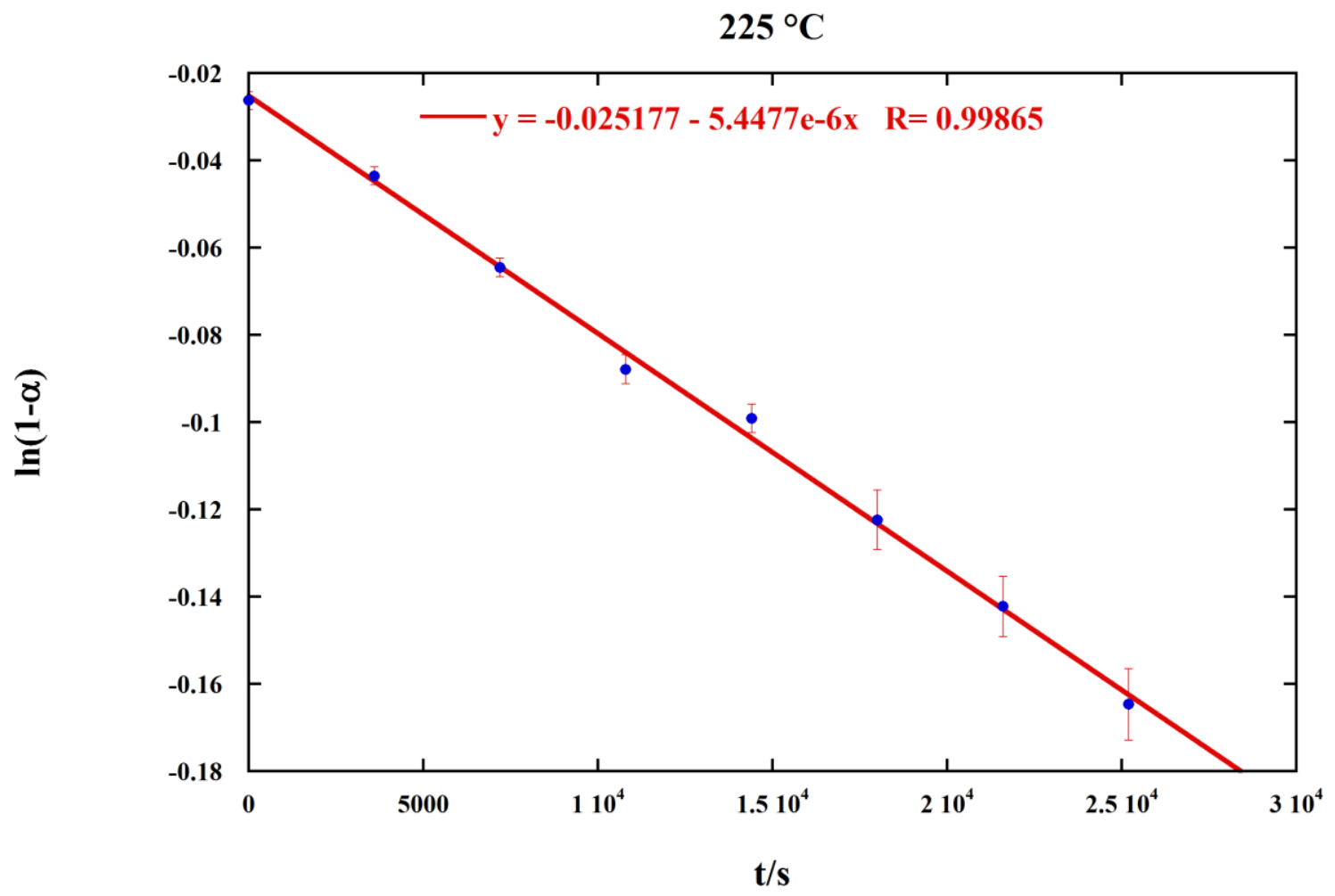

Fig. S4 


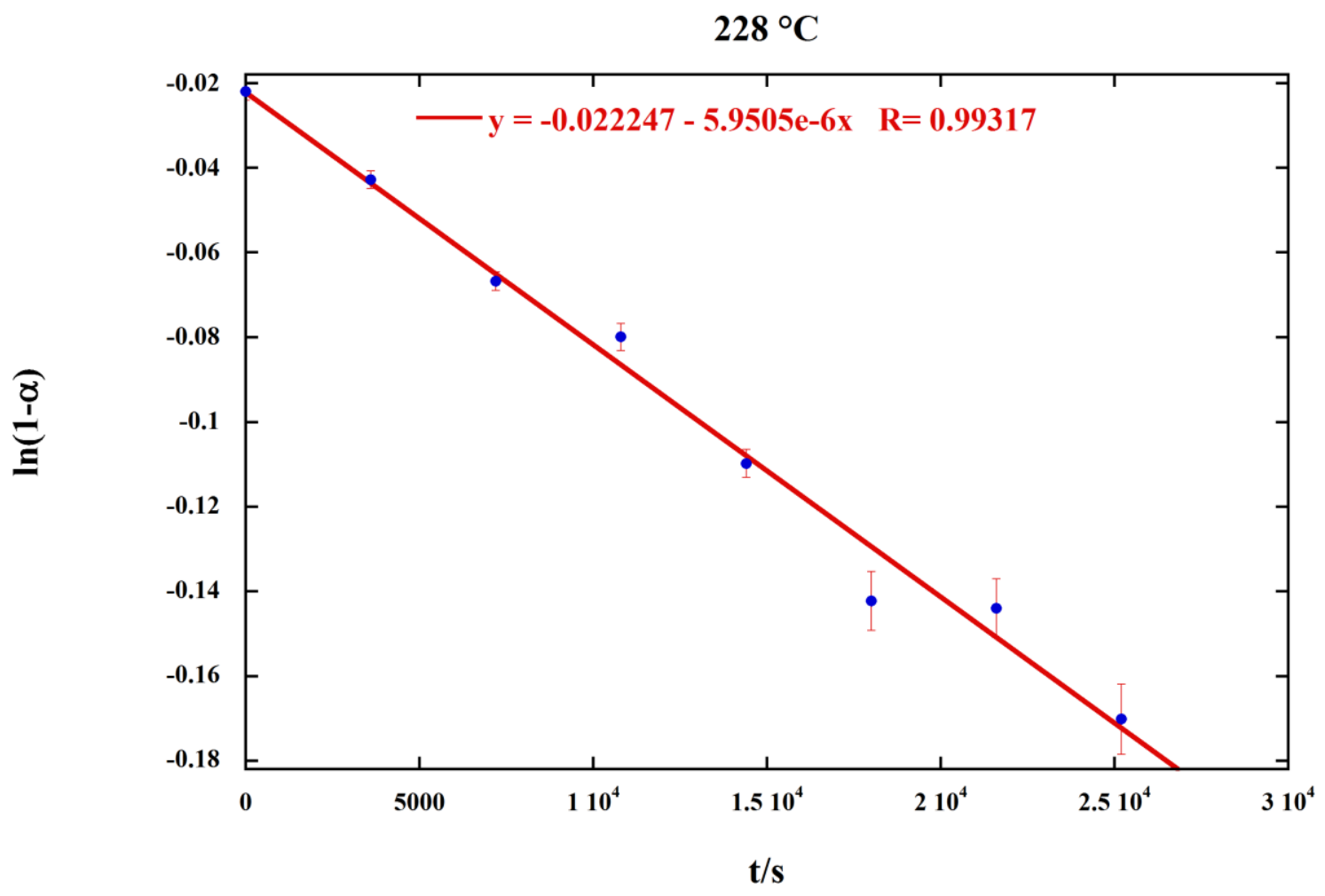

Fig. S5

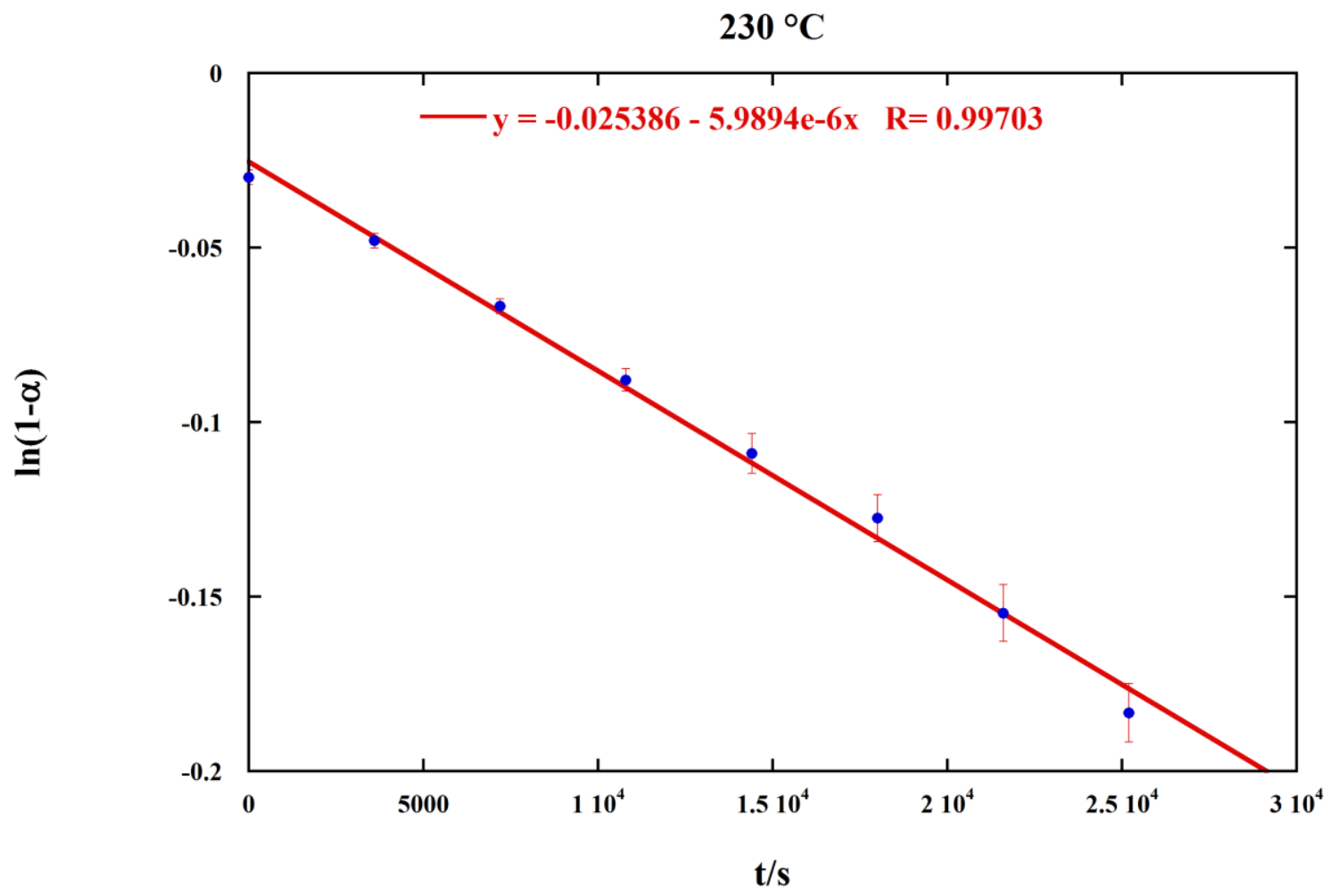

Fig. S6 


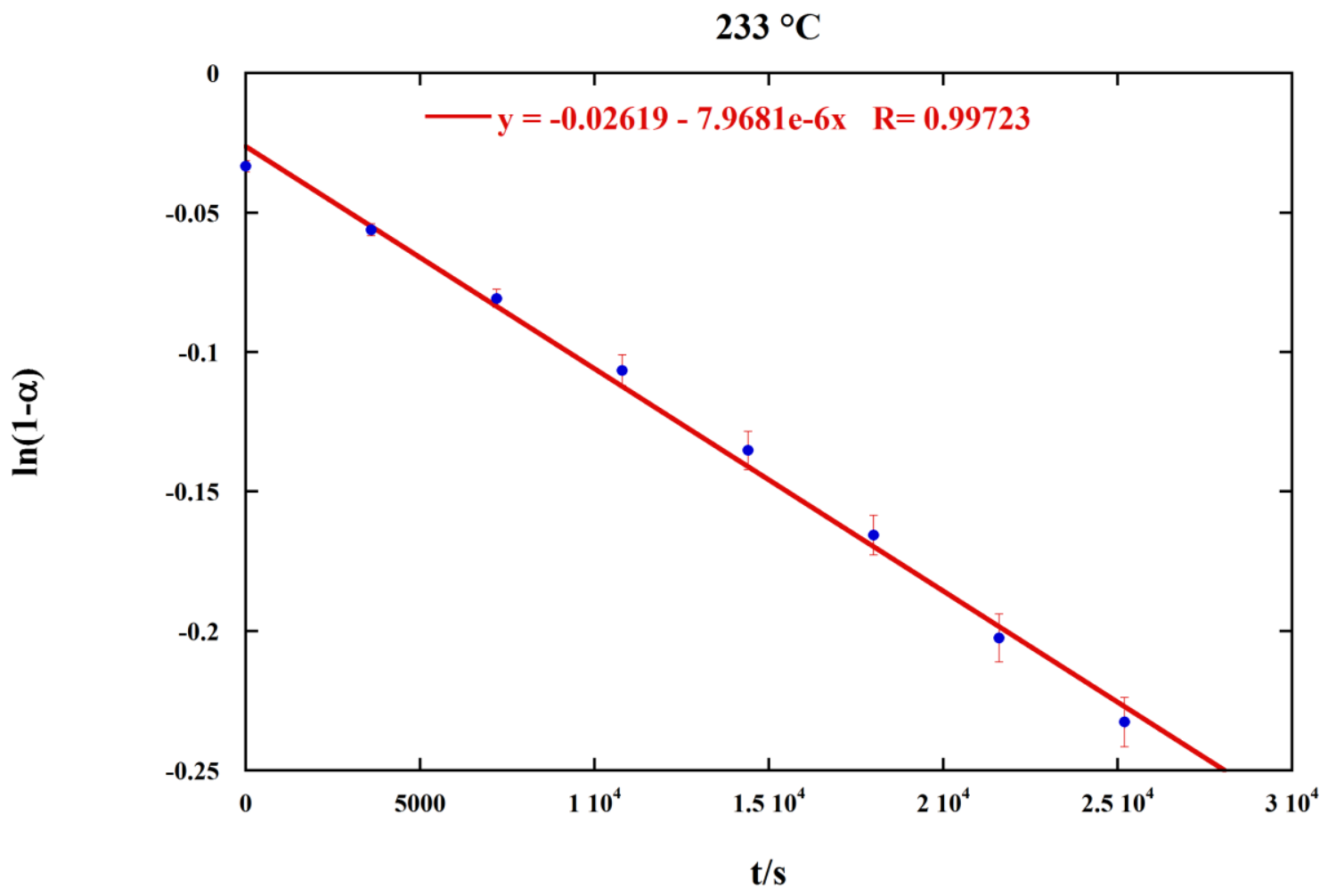

Fig. S7

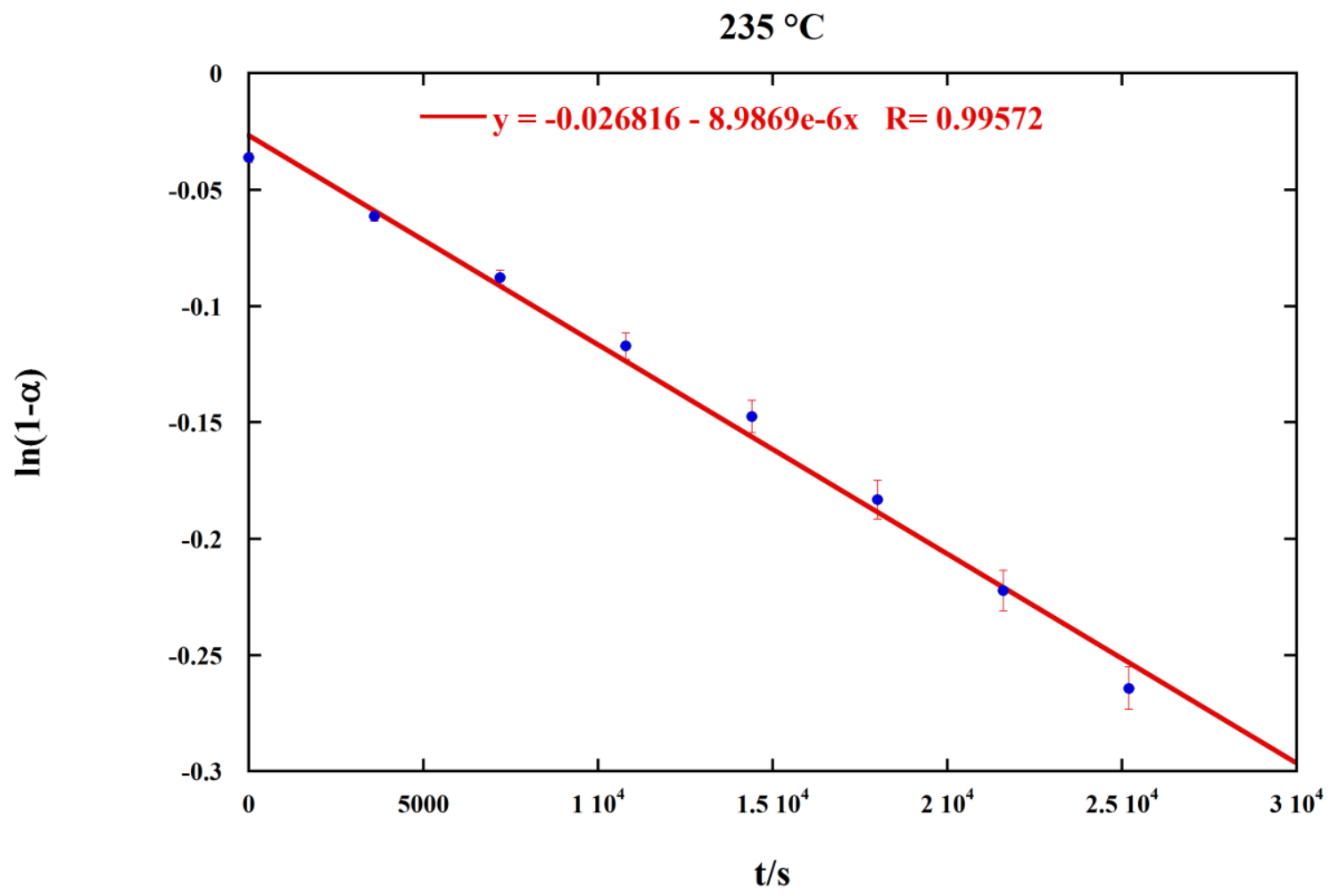

Fig. S8 

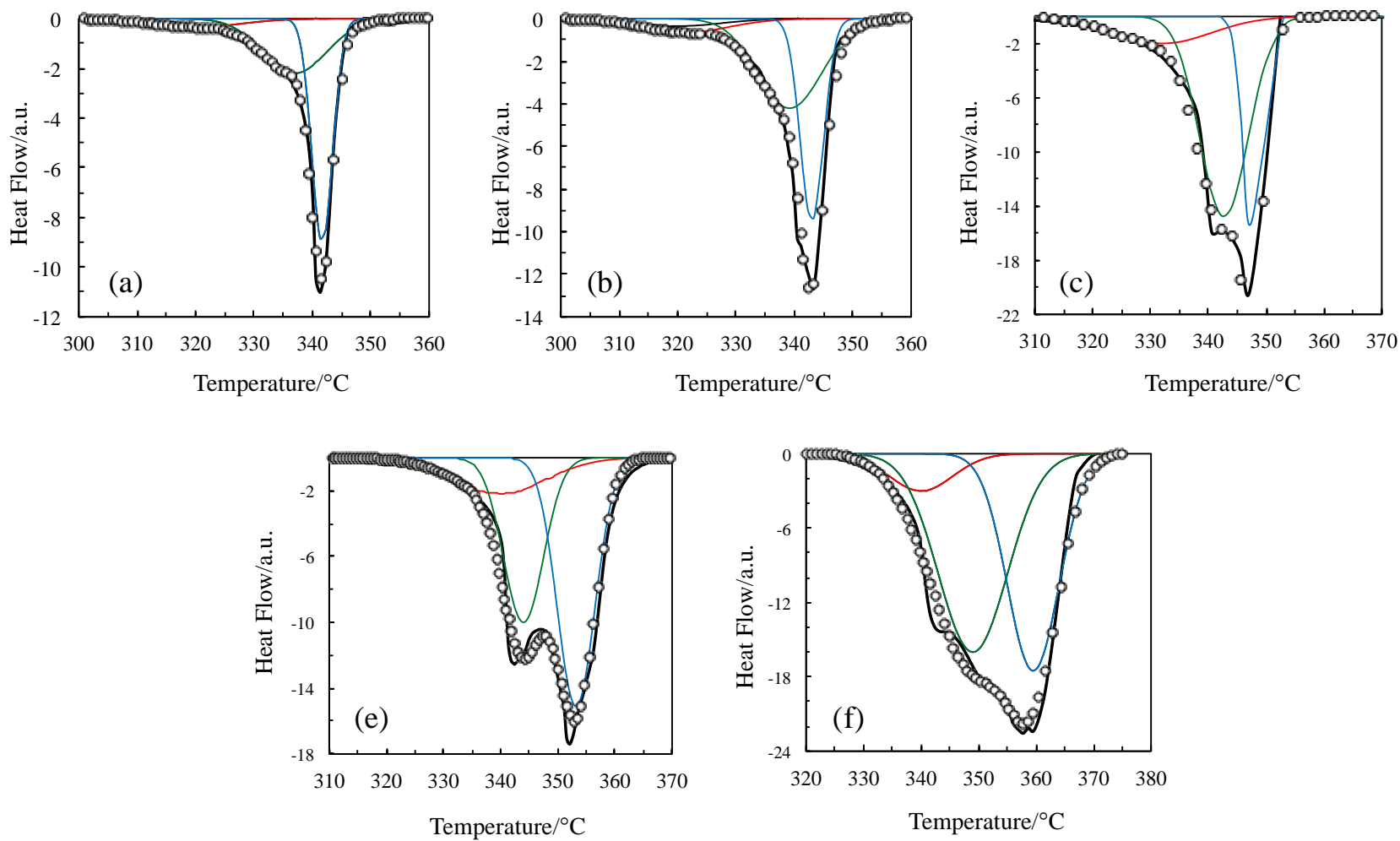

Fig. S9. Deconvolution of the DSC peak recorded at each constant heating rate for FAPI decomposition. 